\title{
A system for the high-throughput analysis of acute thermal avoidance and adaptation in $C$. elegans
}

Andrei-Stefan Lia, Dominique A. Glauser

Department of Biology, University of Fribourg, Fribourg, Switzerland

A

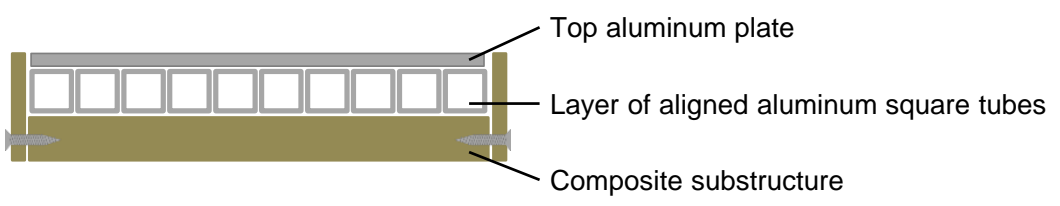

C

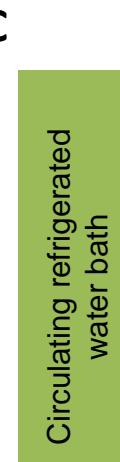

Aluminum square tubes (top view)
B

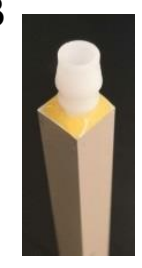

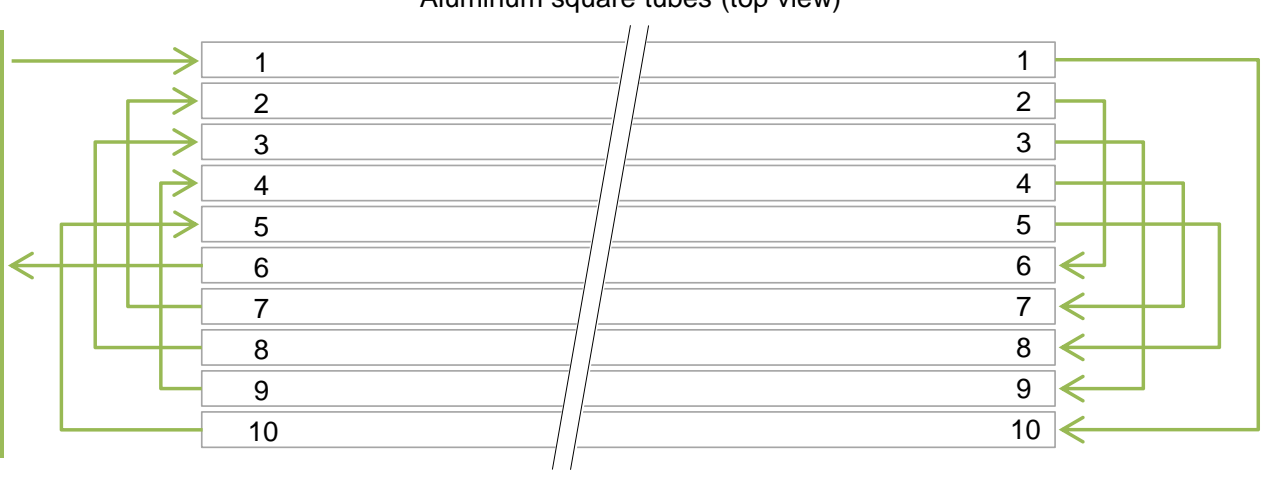

Figure S1. Details of the ThermINATOR cooling module heat exchanger

(A) Side view of the heat exchanger plate

(B) Close up picture of an epoxy-glued fitting at each extremities of the aluminum square tubes.

(C) Tubing connection scheme for the heat exchanger. 
A

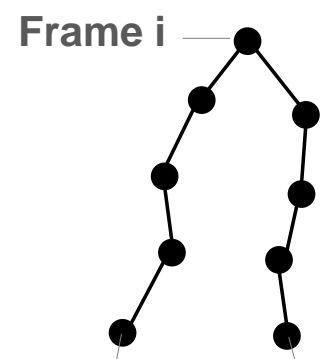

Frame Frame i-4 i+4

B

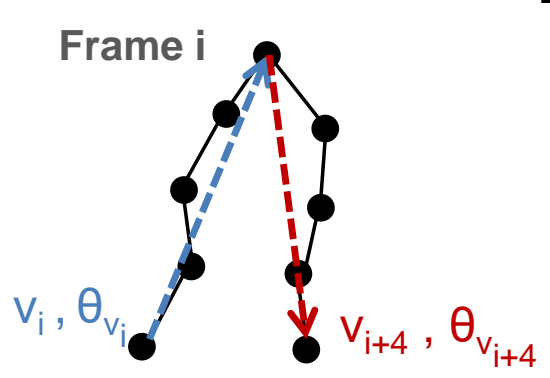

Frame Frame i-4 i+4
C If $\left|\theta_{v_{i}}-\theta_{v_{i+4}}\right| \leq \pi$, then $\theta_{-}$chg $_{i}=\theta_{v_{v_{i}}}-\theta_{v_{i+4}}$ If $\left|\theta_{v_{i}}-\theta_{v_{i+4}}\right|>\pi$, then $\theta_{-} c^{i+4} g_{i}=\left|\theta_{v_{i}}-\theta_{v_{i+4}}\right|-2 \pi$

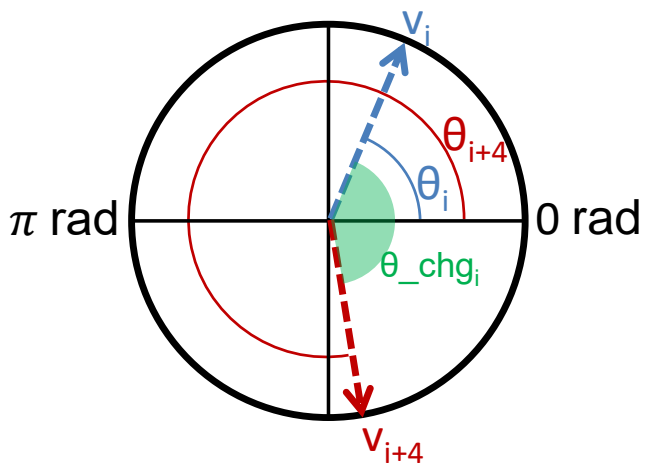

D

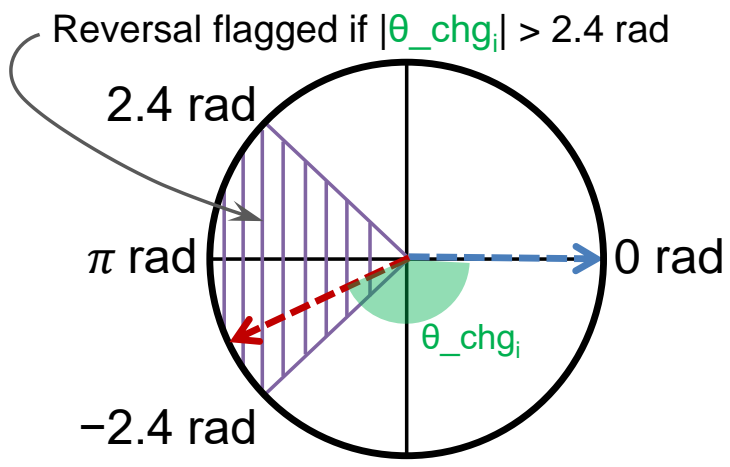

\section{Figure S2. Illustration of the INFERNO reversal flagging criterion}

(A) Schematic showing the xy coordinates of the smoothed trajectory of a tracked worm center-of-mass during nine consecutive frames. The depicted trajectory is centered around frame i and shows the four antecedent coordinates (down to frame i-4) and the four subsequent coordinates (up to frame $i+4$ ).

(B) Illustration of the locomotion vector definition at frame i, used to determine if a reversal flag should be associated to this frame. $V_{i}$ is a vector drawn from coordinates at frame i-4 to coordinates at frame i. $V_{i+4}$ is a vector drawn from coordinates at frame i to coordinates at frame $V_{i+4}$.

(C) Illustration of the angles $\theta_{v_{j}}$ and $\theta_{v_{i+4}}$ taken by the vectors $V_{i}$ and $V_{i+4}$, respectively, in the reference system and of the angle change $\left(\theta_{-} \mathrm{chg}_{\mathrm{i}}\right.$, in green). The angle change calculation formula is depicted.

(D) A reversal event was flagged if the absolute value of the angle change at frame $\mathrm{i}$ was greater than 2.4 radians. This corresponds to the purple hatched area in the figure. 
Number of worm tracks with at least 1 reversal flag Number of worm tracks with 0 reversal flag Total number of worm tracks $\%$ worms reversing in the corresponding $4 \mathrm{~s}$ interval

\begin{tabular}{|r|r|r|r|r|r|}
\hline 24 & 25 & 25 & $94 \ldots$ & 23 \\
\hline 125 & 127 & 129 & $51 \ldots$ & 123 \\
\hline 149 & 152 & 154 & $145 \ldots$ & 146 \\
\hline 16.1 & 16.4 & 16.2 & $64.8 \ldots$ & 15.8 \\
\hline
\end{tabular}

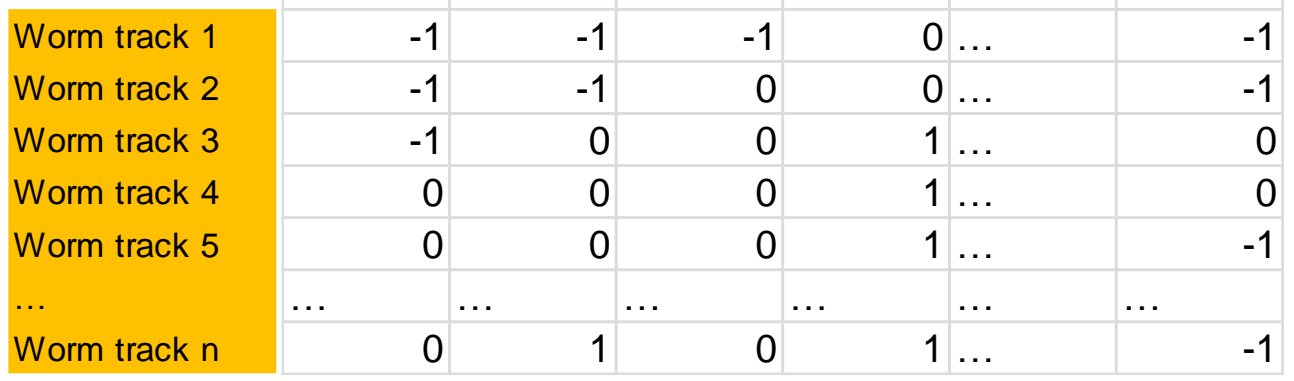

Figure S3. Illustration and explanation of the INFERNO output file Sample INFERNO output tabular .txt file and legends (orange text box). Each column presents data binned over $4 \mathrm{~s}$ intervals, from bin 1 (first 4 seconds of the experiment) to bin $z$ (last four seconds of the experiment). The first four rows provide population level reversal data. Each subsequent row shows behavioral information for an individual worm track, from worm track 1 to worm track $n$, with the following scoring scheme: Worm not tracked $=-1$; Worm tracked, no reversal flagged $=0$; Worm tracked, at least 1 reversal flagged in the bin $=1$. The size of each output file depends on the duration of the experiment (number of columns) and on the number of worm tracks (number of rows). 


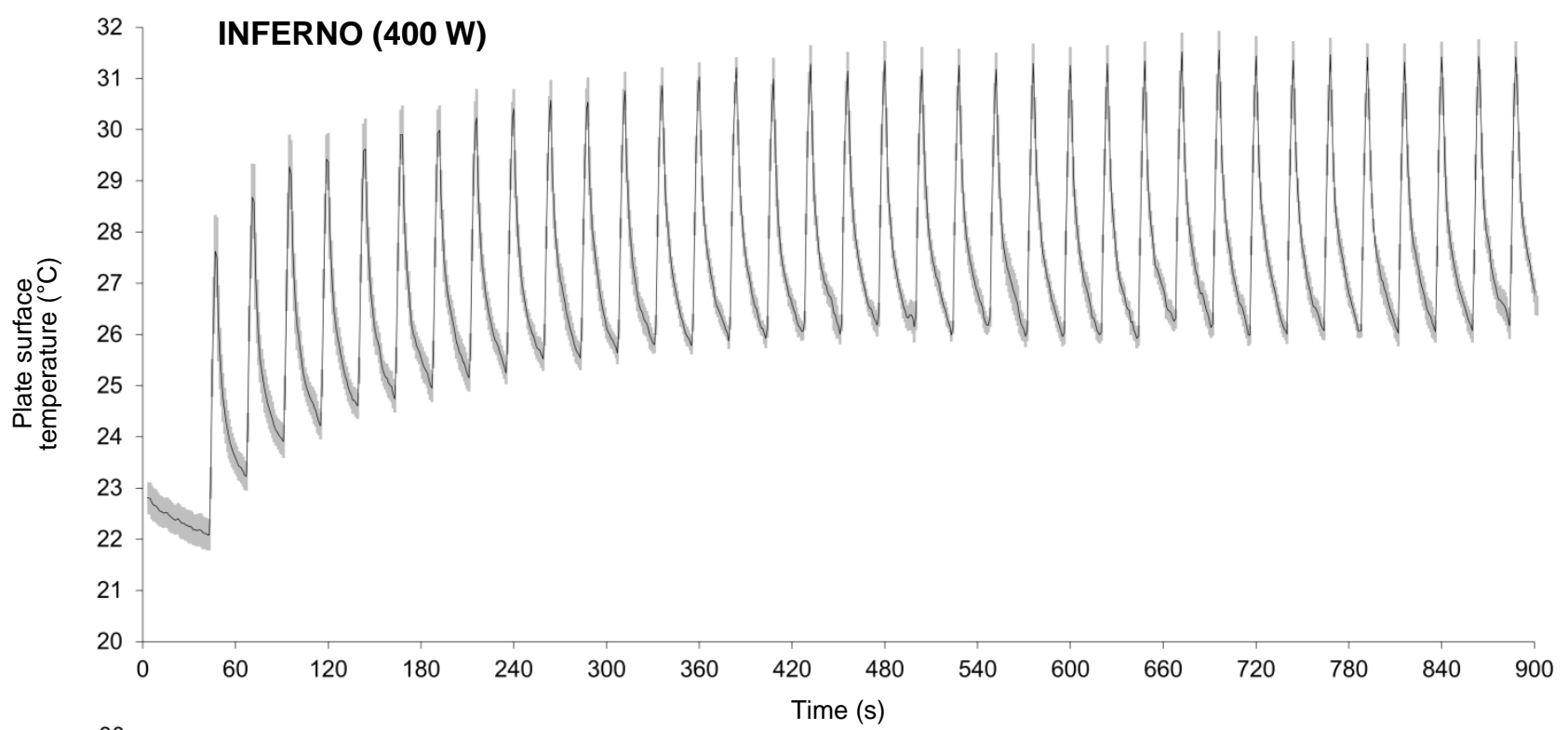

B

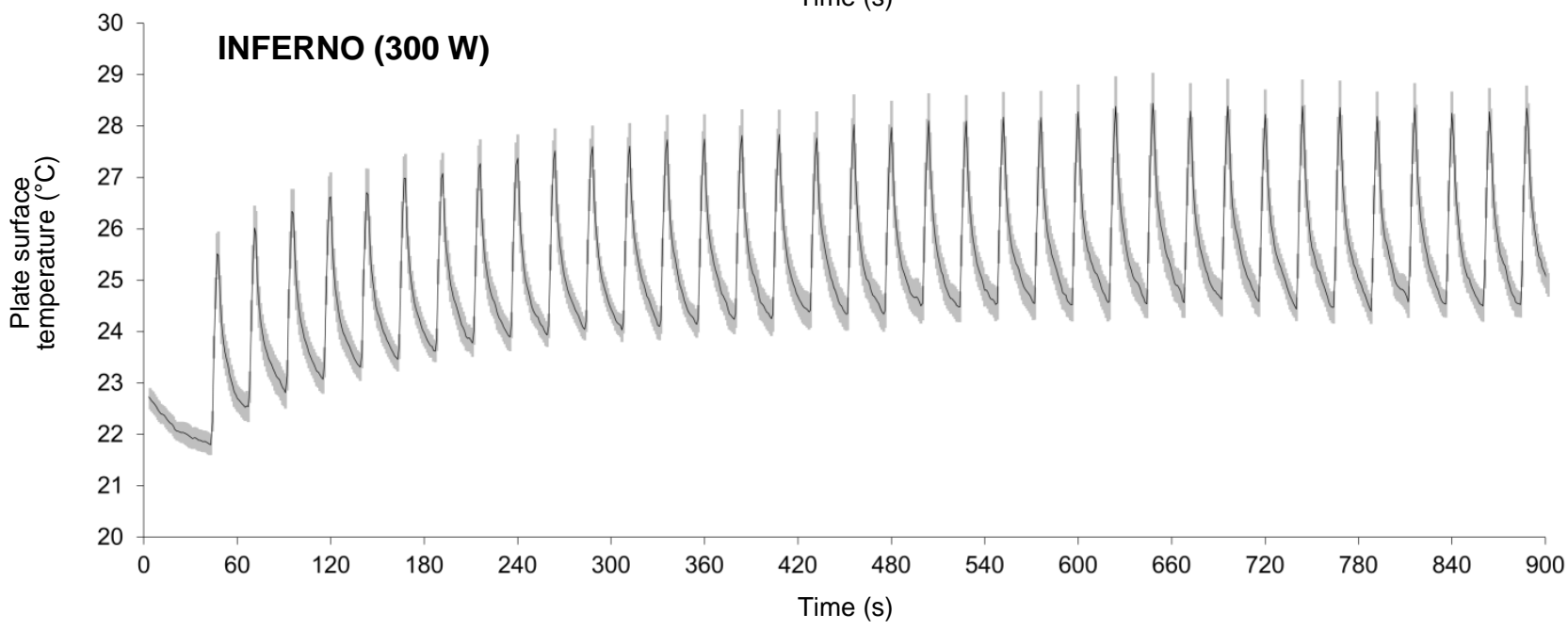

C

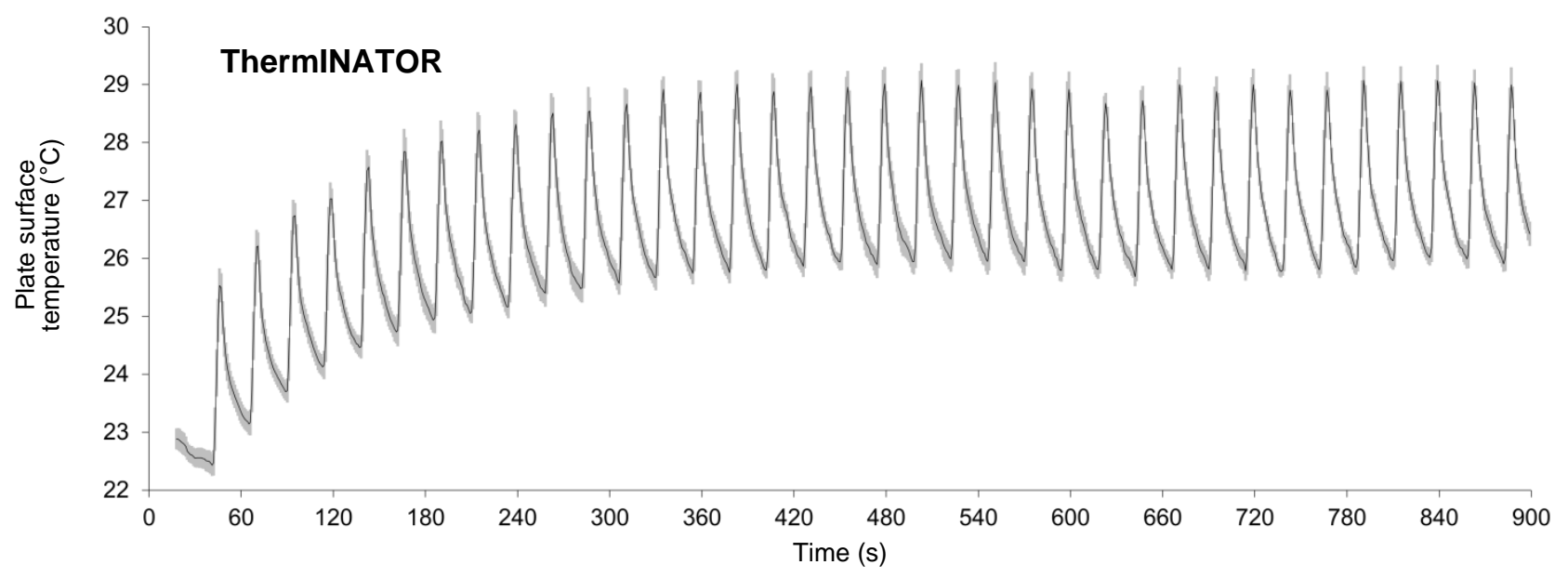

Figure S4. Thermal profiles during adaptation protocols with the INFERNO and ThermINATOR systems

Temperature recorded at the surface of NGM Petri dishes during the first 15 minutes of the adaptation protocols reported in Fig. 6, using a heating power of $400 \mathrm{~W}(\mathbf{A})$ or $300 \mathrm{~W}(\mathbf{B})$ in the INFERNO system, and in Fig. 4C and Fig. 7B with the ThermINATOR system (C). In each situation, the overall temperature rose during the first ten stimuli and plateaued thereafter. The rate of thermal change during each stimuli was remarkably constant. 
A
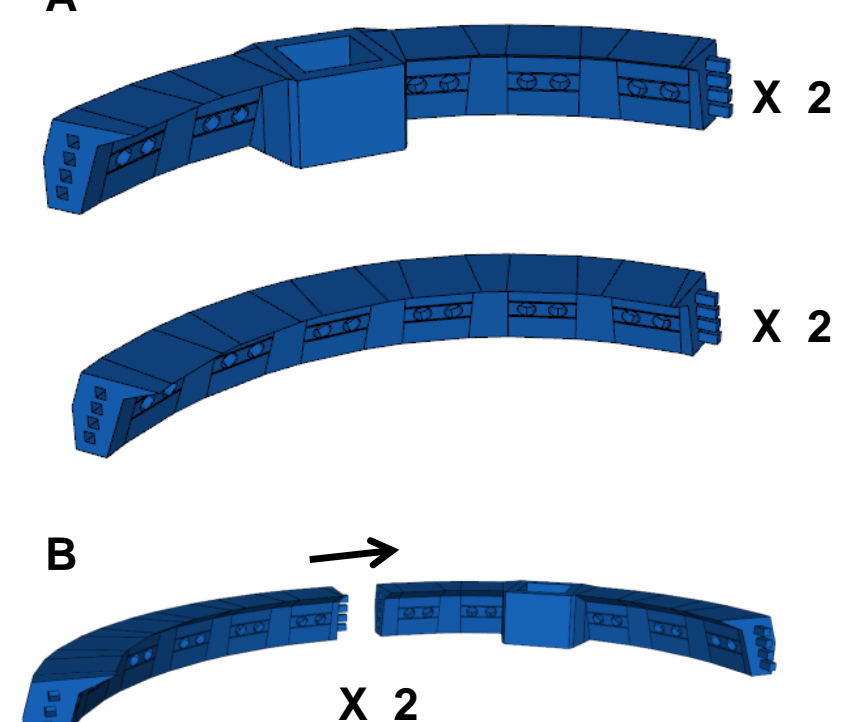

X 2

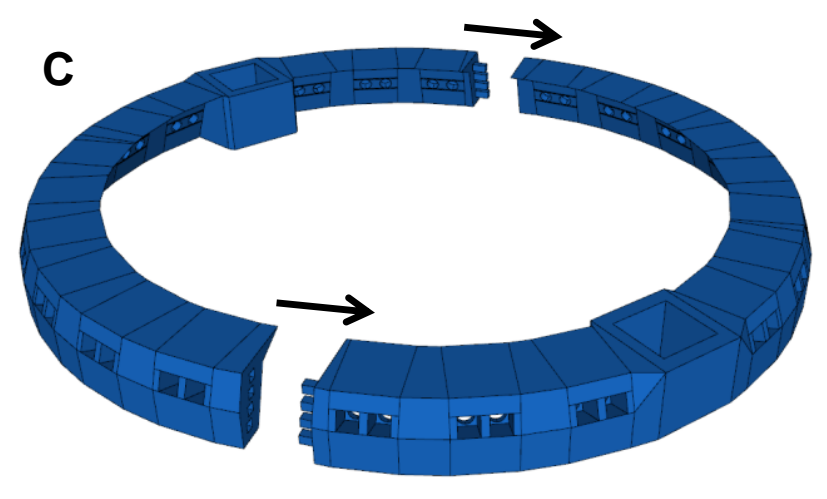

Figure S5. Visual assembly guide for the blue light illumination ring

(A) Model of the two part types obtained by 3D-printing the

INFERNO_LED_ring_segmented.stl file; Both parts come in two copies.

(B) Take one of each part type and assemble as shown. Repeat with the remaining two parts.

(C) Join the two halves of the ring obtained in (B). 
A

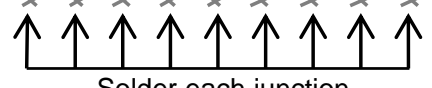

Solder each junction

B

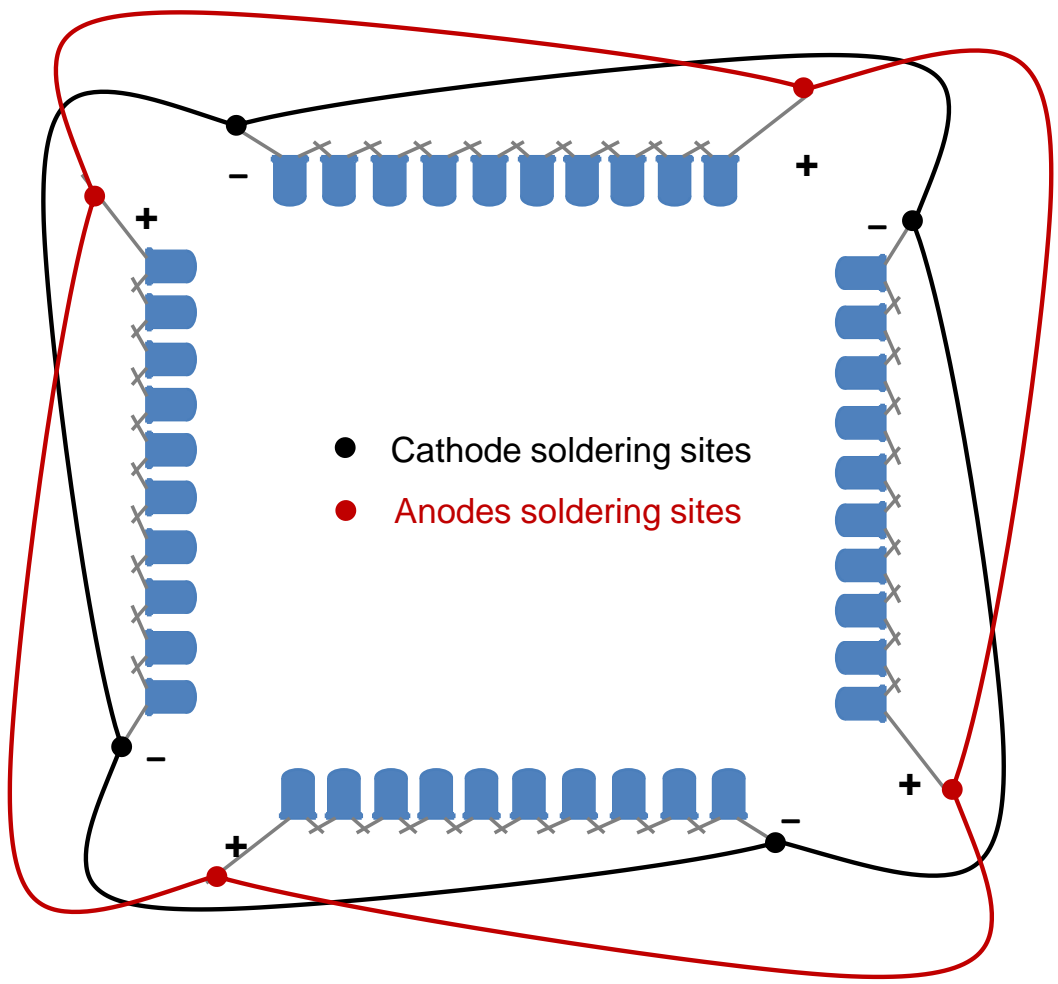

Figure S6. Visual assembly guide for the blue light LED circuit (A) Bend the shown anodes and cathodes, forming 9 junctions. Individually solder each junction.

(B) Lay out a circular wire overlapping the four terminal anodes/cathodes, soldering the anodes together, then the cathodes together. 